$17^{\text {th }}$ International Congress of Metrology, 08003 (2015)

DOI: $10.1051 /$ metrology $/ 201508003$

(C) Owned by the authors, published by EDP Sciences, 2015

\title{
Système innovant non-intrusif de mesures haute température pour le fluide et la paroi de conduites
}

\author{
Mario ARENAS ${ }^{1 \mathrm{a}}$, Didier BOLDO ${ }^{1}$, Sylvain BLAIRON ${ }^{1}$, Laurent ULPAT ${ }^{1}$ \\ ${ }^{1}$ EDF R\&D, EDF Lab Chatou 6 quai Watier 78101 Chatou, France
}

\begin{abstract}
EDF is a major operator of Nuclear Power Plants (NPP). EDF R\&D conducts researches on nonintrusive techniques able to measure temperature inside and outside a pipe. In order to test two of them, a trial was conducted on AREVA JSPM test loop. This loop can create the primary loop conditions $\left(155 \mathrm{bars}, 290^{\circ} \mathrm{C}\right.$, $22000 \mathrm{~m} 3 / \mathrm{h}$ ). The two tested systems were: a fiber optic based for surface temperature measurement and an ultrasonic device for water temperature measurement. They have been used in heterogeneous area, due to the cooling system. Both systems have been compared to the loop internal measurement system and to classical thermocouples for surface temperature. The first system is an optical fiber placed under the heat insulator. Temperature is measured all along the full length of the fiber, with a $0.25 \mathrm{~m}$ spatial step thanks to a DTS (Distributed Temperature Sensor) using Raman effect. This system has successfully provided surface temperature cartography of the mixing zone. The second system uses ultrasonic probes to compute the speed of sound in the water. As pressure is known, temperature can be calculated from this celerity. This system enables the non-intrusive measurement of the water temperature in industrial conditions, and is especially effective when temperature is heterogeneous, as it provides a temperature average. Results show a very good agreement between this measurement and internal probes.
\end{abstract}

\section{CONTEXTE}

EDF exploite en France un parc de 58 réacteurs nucléaires, et est un acteur majeur de ce secteur dans le monde. Une particularité de ce type d'industrie est le niveau élevé des exigences relatives aux équipements nécessaires pour garantir la performance et la sûreté des centrales.

Pour le respect de ces exigences, EDF travaille régulièrement sur l'amélioration de l'instrumentation thermohydraulique, élément indispensable pour le contrôle et la surveillance des centrales.

La principale contrainte lors du développement de nouvelles instrumentations est l'éventuelle modification du matériel existant. En théorie, aucune modification matérielle n'est envisageable et quand elle est indispensable, une démonstration doit être réalisée pour prouver l'innocuité de cette modification.

EDF s'est, de ce fait, orienté vers les instrumentations non intrusives qui par définition n'impactent pas l'intégrité du composant sur lequel elles sont installées.

Deux technologies font l'objet d'études approfondies depuis plusieurs années : les ondes ultrasonores (UT) et la fibre optique (FO). Les deux technologies donnent accès à la grandeur physique température. Pour la FO, il s'agit de la température sur la paroi extérieure (température de peau) et pour les UT, de la température moyenne sur une corde du fluide (eau) traversant la conduite.
Ces deux technologies ont suscité de nombreuses investigations mais la particularité de cette nouvelle étude concerne leur installation dans un environnement semiindustriel (boucle thermohydraulique) dans des conditions représentatives de celles du circuit primaire d'une centrale nucléaire $\left(300^{\circ} \mathrm{C}, 155 \mathrm{bar}, 25000 \mathrm{~m}^{3} / \mathrm{h}\right)$ et les performances métrologiques associées. Ce document a pour but de présenter les aspects technologiques et métrologiques des systèmes de mesure de la température mis en place sur un banc semi-industriel.

\section{LA BOUCLE D’ESSAIS}

\subsection{Description de la boucle}

Le centre d'essais de JSPM à Maubeuge est un centre d'essai dédié à la qualification des Groupes MotoPompe Primaire (GMPP) pour les centrales nucléaires. Son objectif est de caractériser les pompes en fonctionnement, dans des conditions équivalentes aux conditions réelles. Le circuit est donc prévu pour fonctionner à 155 bars, $290^{\circ} \mathrm{C}$, avec un débit jusqu'à $28000 \mathrm{~m}^{3} / \mathrm{h}$.

Cette boucle d'essai est représentative des conditions réelles d'un circuit primaire de centrale nucléaire, hors radiation. La boucle d'essai dispose de différents capteurs

a mario.arenas@edf.fr 
permettant son contrôle et sa surveillance, notamment de capteurs spécifiques pour la réalisation des courbes des pompes (mesure du delta $\mathrm{P}$ pompe et du débit).

\subsection{Les essais}

\subsubsection{La zone instrumentée}

La zone choisie pour les mesures de température de peau est la zone de mélange entre l'eau principale et l'eau de refroidissement. En effet, la pompe transmet l'intégralité de sa puissance à l'eau, qui la dissipe en frottements et finalement en chaleur. Par conséquent, la température de la boucle augmente rapidement et un refroidissement est donc nécessaire. Ce refroidissement est réalisé par injection dans la partie basse, en amont de la pompe, d'une eau plus froide de $30^{\circ} \mathrm{C}$ environ. C'est cette zone qui a été choisie pour être instrumentée, car elle présente des hétérogénéités de températures du même ordre de grandeur que la branche chaude d'une centrale nucléaire, à des températures proches.

\subsubsection{Mesures de référence}

Afin de disposer de mesures de comparaison pour les températures de peau par FO, dix thermocouples ont été positionnés sur la surface de la canalisation, dans des zones d'intérêt. Chaque thermocouple a été fixé à la surface et aux fibres proches à l'aide d'un scotch haute température. La Figure 1 présente les positions des 10 thermocouples. Chaque thermocouple, en plus d'être une mesure commune avec la fibre, a été positionné avec un objectif : -TC1 : température du fluide de refroidissement ;

-TC3 et TC7 : température du fluide chaud, avant mélange ;

-TC8, TC10, TC9 et TC5 : mesure de la température de la zone de mélange, au fur et à mesure du mélange ;

-TC2, TC4 et TC6 : mesure de 1'extension de la zone de mélange.

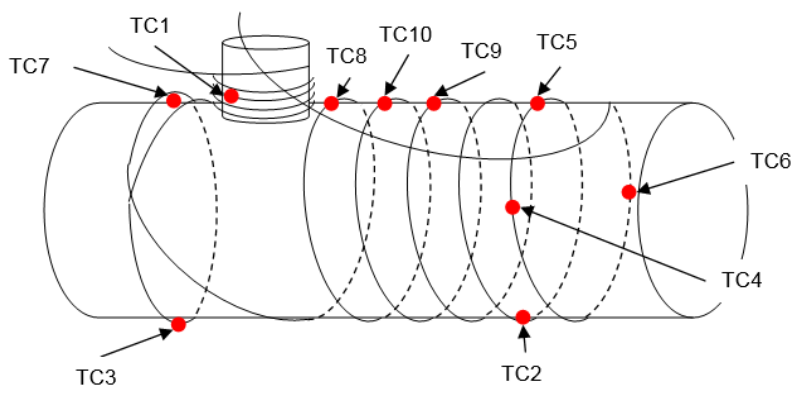

Figure 1. Positionnement des thermocouples (la spirale noire est la FO)

\subsubsection{Le plan d'expérience}

La boucle est remplie et montée en pression. La pompe est ensuite mise en fonctionnement, ce qui échauffe le fluide par frottements, jusqu'à atteindre la température désirée. Les mesures sont réalisées dès que la boucle est en eau, mais elles ne sont pertinentes que lorsque la boucle monte en température.

\subsection{Fibre optique}

\subsubsection{Procédé de mesure de la mesure de température par effet Raman}

Un système de mesure de la température distribué par effet Raman est capable de mesurer la température tout le long d'une fibre optique, avec un certain pas spatial (voir [1] et [2]). Le principe physique est la diffusion inélastique des photons par le milieu dans lequel ils se propagent. Cette perte d'énergie se traduit par une diffusion dans une longueur d'onde différente de la longueur d'onde d'émission. Pour la mesure de température par effet Raman, une impulsion laser monochromatique est envoyée dans une fibre optique. Celle-ci diffuse alors, par effet Raman, aux fréquences Stokes et anti-Stokes. Le système mesure alors, à tout instant, la puissance rétrodiffusée au sein de la fibre, à ces deux fréquences. Le rapport entre ces deux puissances est directement lié à la distribution de Boltzmann et donc à la température. L'instant de retour de l'impulsion permet de calculer la distance de la zone mesurée.

\subsubsection{Choix des câbles}

Les câbles ont été sélectionnés en fonction de plusieurs critères. Ils devaient parfaitement résister aux conditions de fonctionnement de la boucle d'essai (environ $290^{\circ} \mathrm{C}$ ), être capables de résister aux conditions d'une branche chaude d'un REP, être résistants et faciles à poser. Suite à des essais réalisés précédemment, EDF R\&D a retenu 4 câbles en inox de $30 \mathrm{~m}$, réalisés par sertissage de morceaux de 2 mètres, contenant :

1. une fibre optique multimode gainée cuivre ;

2. une fibre optique multimode gainée or ;

3. une fibre optique multimode gainée or et une fibre optique monomode gainée or (non utilisé) ;

4. une fibre optique monomode gainée or (non utilisé).

\subsubsection{L'installation}

Les fibres sont installées dans la zone de mélange de température induite par le système de refroidissement (voir 2.1.1).

Une glacière a été utilisée en tant qu'enceinte thermostatée : 2 à 3 mètres de câbles FO sont enroulés à l'intérieur afin d'obtenir un plateau de température qui servira à calibrer l'interrogateur Raman

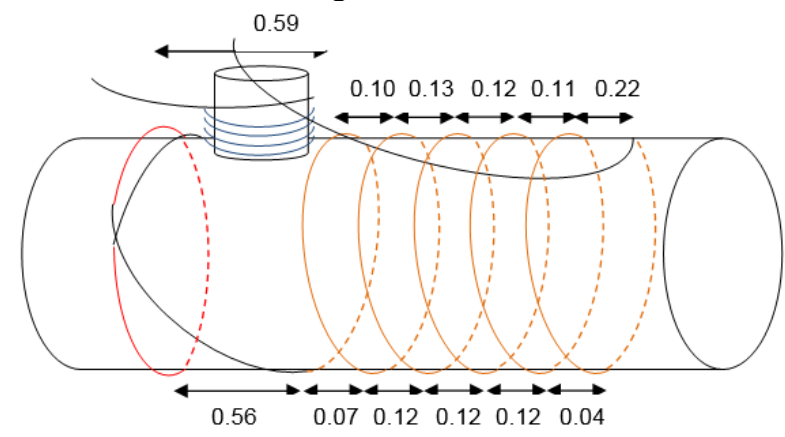

Figure 2. Géométrie de pose de la fibre (distance en m) 
Les câbles ont été installés en spirale côte à côte sur la tuyauterie afin de pouvoir les comparer les différentes mesures de température point à point entre elles.Figure 2. Les câbles de mesure sont reliés à l'instrument par des rallonges en fibres standards. Ils traversent la zone thermostatée (glacière) avant d'être enroulés autour de l'amenée d'eau de refroidissement, zone homogène de température «froide » (en bleu sur la Figure 2). Puis, ils s'enroulent autour de la canalisation principale, en amont de la zone de mélange, zone homogène de température « chaude » (en rouge sur la Figure 2). Puis, les câbles spiralent autour de la canalisation principale, après la zone de mélange, afin de capturer celle-ci (en orange sur la Figure 2). Enfin, ils reviennent le long de la génératrice supérieure avant de sortir de la zone de mesure.

Tout comme les thermocouples, les câbles fibre optique permettent de réaliser une mesure de surface par contact.

\subsubsection{Exploitation des données}

Les systèmes de mesure de température par effet Raman envoient une impulsion laser monochromatique et mesurent la puissance rétro diffusée au sein de la fibre, à deux fréquences appelées pic Stokes et anti-Stokes. L'instant de retour de l'impulsion permet de calculer l'abscisse de la zone mesurée. A tout instant $t$ et en tout point d'abscisse $l$, le rapport entre l'énergie de ces deux pics est lié à la température de la fibre par la formule suivante :

$$
T(l, t)=\frac{\gamma}{\ln \left(\frac{P_{\text {Stokes }}(l, t)}{P_{\text {anti-Stokes }}(l, t)}\right)-\int_{0}^{l} \alpha(x, t) d x+C(t)}
$$

Avec

- $l$ : abscisse curviligne le long de la fibre, avec 0 au début de la ligne de mesure (l'appareil contient une certaine longueur de fibre en interne, dont l'abscisse est négative); - $T$ : température moyenne de la fibre $(\mathrm{K})$ à l'instant $t$ et à l'abscisse $l$;

- $\gamma$ : dépend théoriquement de la distribution des états quantiques de la fibre, mais est considéré comme constant pour un appareil donné ;

- $\alpha$ : atténuation différentielle entre les deux longueurs d'onde Stokes et anti-Stokes au point d'abscisse $x$

- $C$ : coefficient lié à l'écart de gain du système de mesure entre les longueurs d'onde des deux pics Stokes et antiStokes.

Les paramètres $\gamma$ et $C$ sont fournis par l'instrument.

Le paramètre $\alpha$, lui, dépend de la fibre utilisé et doit donc être évalué sur la ligne de mesure. Plusieurs méthodes existent pour cela :

- Il est supposé connu et constant, et est intégré comme paramètre ;

- Il est recalé grâce à une zone homogène de température connue (ou mesurée) ;

- Il est éliminé grâce à une mesure depuis les eux extrémités de la fibre (mode double-end).

Dans ce dernier cas, la formule utilisée est légèrement différente :

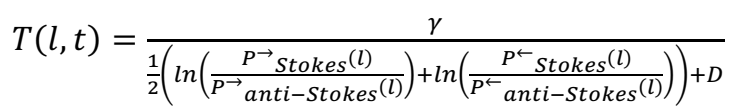

Nous utilisons cette dernière solution pour le câble 1 , car elle est plus rigoureuse (élimination d'un paramètre) et modifie peu notre installation. Cependant, afin d'être en mesure de superposer les deux sens de lecture, il est nécessaire de fournir à l'instrument la longueur totale de fibre. Sur le terrain, cette détermination précise peut être difficile à réaliser.

\subsection{Ultrasons}

\subsubsection{Procédé de mesure}

Le principe de mesure de la température par ultrason est de de mesurer la célérité du son dans l'eau. Cette célérité dépend de la température et de la pression. Comme, dans les conditions d'essai, la pression est connue, la mesure de la célérité permet de mesurer la température (voir Figure 3).

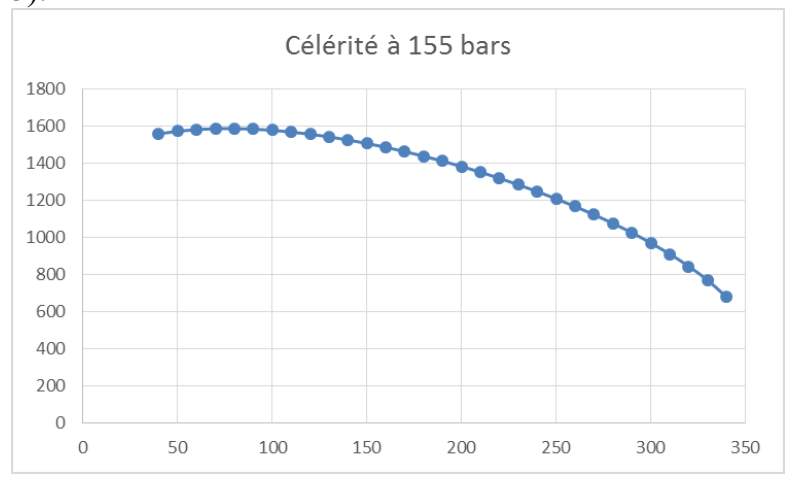

Figure 3. Célérité de l'eau $(\mathrm{m} / \mathrm{s})$ selon la température $\left({ }^{\circ} \mathrm{C}\right)$

Les capteurs ultrasonores sont placés sur la tuyauterie. Ils envoient une onde ultrasonore qui se propage à travers l'eau, se réfléchit sur l'interface eau-acier et revient vers le capteur. La distance parcourue dans l'eau étant connue, la mesure du temps de vol de cette onde permet de remonter à la célérité de l'eau et donc à sa température. Contrairement à la fibre optique, la température appréhendée ici est bien celle du fluide et non celle de la tuyauterie.

La sensibilité de ce type de mesure est très variable selon les conditions du fluide (pression et température). Dans les conditions du circuit primaire d'une centrale nucléaire, la célérité varie de manière importante en fonction de la pression et de la température et est donc un bon candidat pour cette mesure.

\subsubsection{Les capteurs UT, le couplant et le système de fixation}

Les capteurs ultrasons utilisés sont spécialement conçus pour résister à la température et peuvent donc être placés 
directement au contact de la tuyauterie, sous le calorifuge. Le point délicat est le couplage entre le transducteur ultrasonore et la canalisation. En effet, l'onde ultrasonore doit passer du transducteur au métal de la canalisation puis à l'eau avec le moins de pertes possibles. Classiquement, des gels sont utilisés, mais ceux-ci sont peu résistants à la température dans la durée. Par conséquent, le couplage a été réalisé à l'aide d'une fine feuille métallique. Cela implique une force d'appui importante, qui nécessite un système de fixation spécifique.

\subsubsection{L'installation}

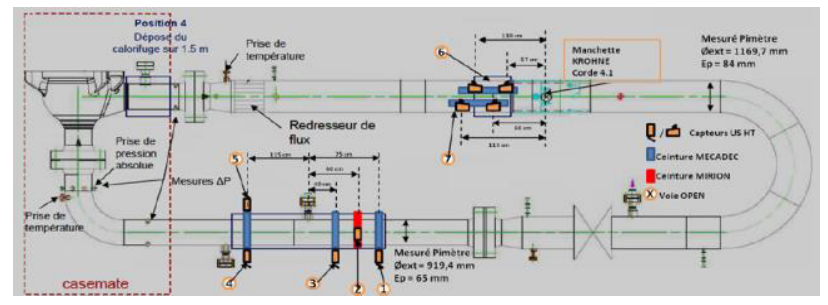

Figure 4 : Zone d'installation des capteurs ultrasons

Les capteurs ont été installés sur la même zone que les fibres optiques (voir Figure 4), en amont et en aval de la zone de mélange du refroidissement. Des essais de couplage ont été réalisés avec de l'or et du plomb. De par leur nature métallique, les feuilles sont peu malléables et nécessitent donc des états de surface de la tuyauterie très lisses pour permettre une bonne transmission du signal. Une reprise des états de surface a été nécessaire afin d'obtenir une qualité de signal satisfaisante.

Par ailleurs, un calorifuge spécialement adapté a été mis en place autour du système de fixation, afin d'éviter de créer une zone de déperdition thermique.

\section{Résultats}

\subsection{Fibre optique}

\subsubsection{Visualisation directe}

Une visualisation simple des données lorsque la canalisation est en température est proposée.

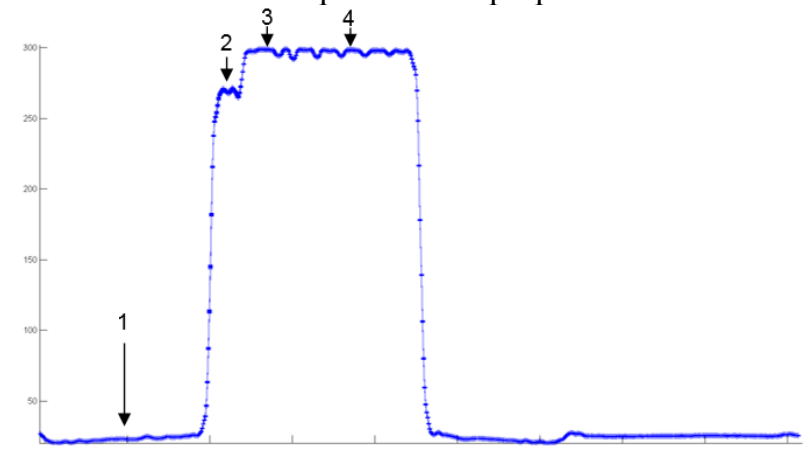

Figure 5. Température le long de la fibre
L'interprétation de la Figure 5 est assez simple, car elle suit le schéma de pose présenté Figure 2 :

1. la zone de fibre à l'air libre mesure la température ambiante : elle va de l'instrument jusqu'à la zone de mesure ;

2. la zone qui entoure la canalisation d'arrivée d'eau de refroidissement ;

3. la zone qui entoure la canalisation principale (chaude) ;

4. les tours de la zone de mélange, les « vagues » représentant l'hétérogénéité de température générée par le mélange des fluides de température différente.

\subsubsection{Données double-end}

En calculant les températures à partir des données en sens direct et inverse (voir Figure 6), il apparaît clairement que les deux mesures sont décalées géométriquement. Or, en mode double-end (voir 2.2.4), le calcul de température utilise ces mesures et fournit donc une température « moyennée » entre les deux sens. La longueur totale de fibre utilisée par l'instrument n'était donc pas correcte et a donc dû être corrigée a posteriori pour obtenir des valeurs de température réalistes.

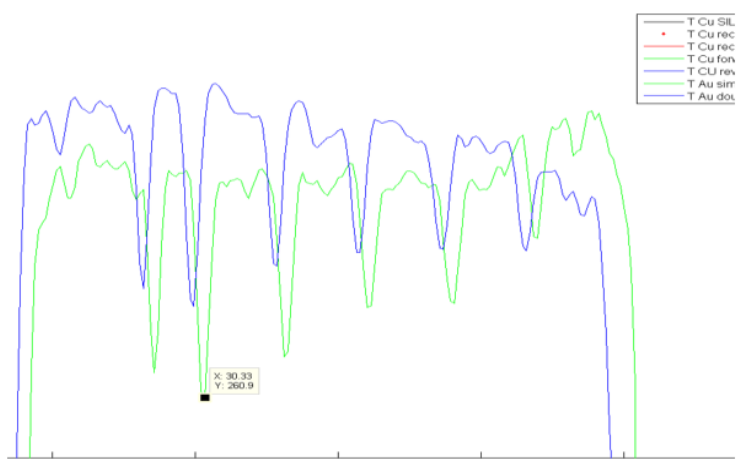

Figure 6. Comparaison des températures calculées dans le sens directe et inverse

Une fois cette correction effectuée, la température est calculée grâce à l'équation (3). La Figure 7 présente la comparaison des trois fibres après correction de la fibre double-end (cuivre) par recalage spatial. Les trois fibres donnent alors le même profil de température, ce qui valide la correction. 


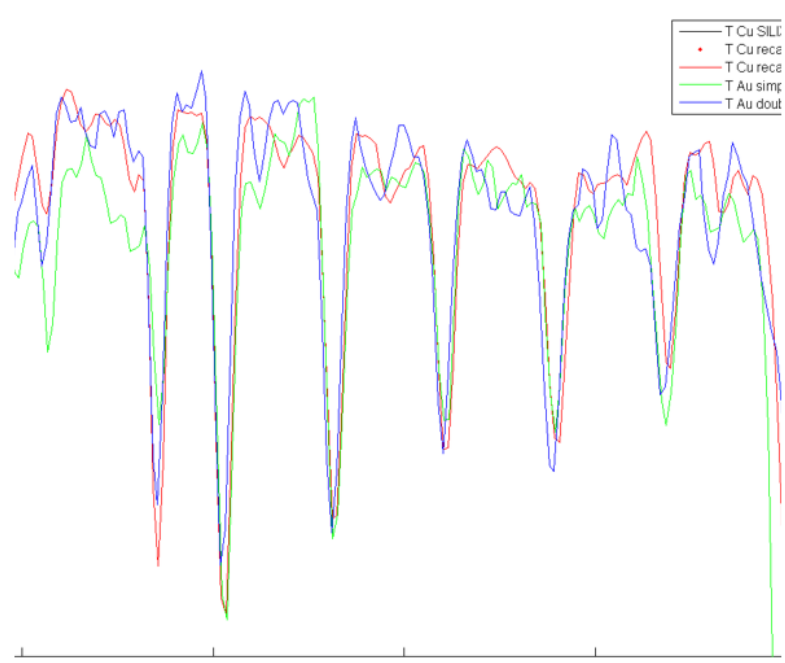

Figure 7. Comparaison des températures entres les trois fibres après recalage spatial

\subsubsection{Comparaison aux autres données}

En repérant manuellement les positions des thermocouples sur la fibre, il est possible de faire une comparaison directe entre les mesures fibre et les mesures par TC. Un recalage temporel (non synchronisation des deux systèmes d'enregistrement) et en valeur (calibrage par un offset) sont appliqués aux données FO. La

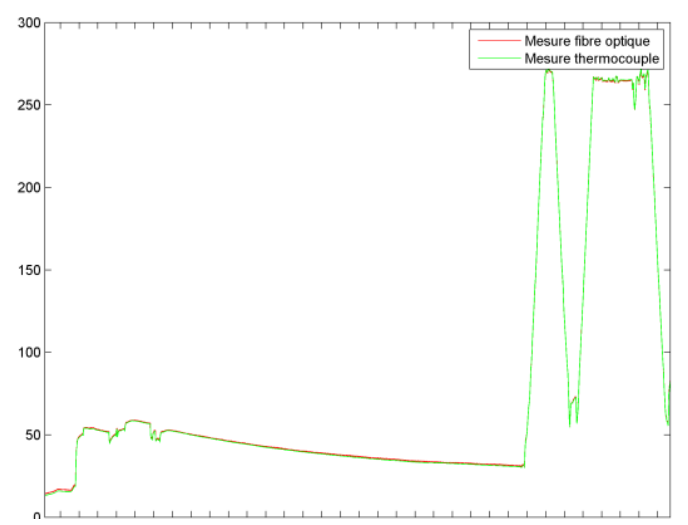

Figure 8a présente cette comparaison, pour la fibre or double. Chaque couleur représente une position, en trait plein par thermocouple, en pointillé par fibre optique.

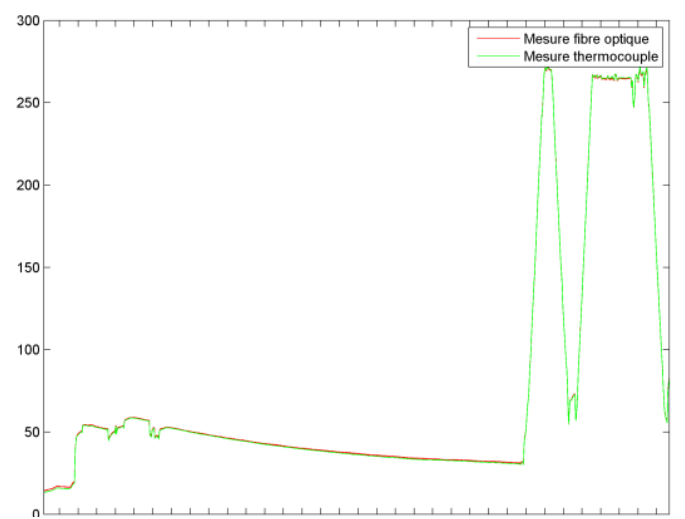

Figure 8b Fibre et thermocouple sur l'ensemble des essais
Il apparaît immédiatement que les mesures sont cohérentes, puisque les variations sont bien similaires entre les deux systèmes.

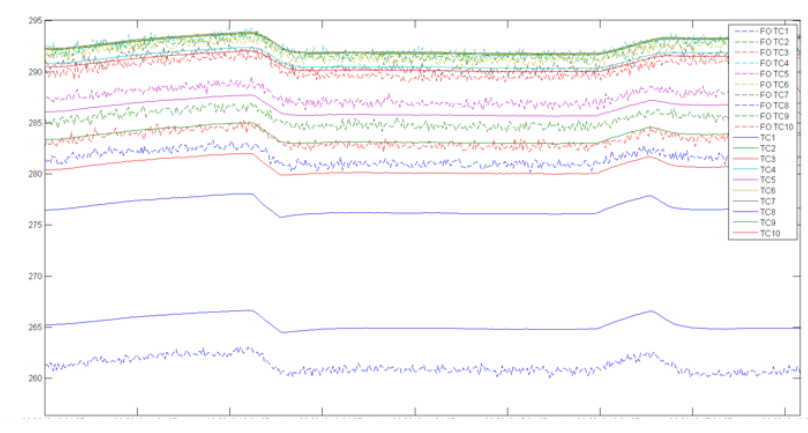

Figure 9. Fibre et thermocouple lors d'une période stabilisée à chaud

La Figure 9 présente un extrait sur une période stabilisée. En variations relatives, on trouve des résultats cohérents avec la physique : le point 1 (voir Figure 1) est le plus froid, suivi dans l'ordre par les points $8,10,9$ et 5 . Les points 3 et 7 sont légèrement $\left(1^{\circ} \mathrm{C}\right.$ ) plus froids que les autres (points 2,4 et 6), alors qu'ils sont en contact avec la partie de la canalisation avant refroidissement, donc normalement la plus chaude. Un explication possible est que la fibre passe très près du bord de l'isolant de ce côté de la boucle, et qu'elle n'est donc pas parfaitement à la température de la canalisation.

« ce qui n'est pas physique » me gêne, mais je n'arrive pas à trouver autre chose. En fait, c'est bien physique puisque ca mesure bien une température entachée du biais dû au bord du calo. Peut-être « ce qui n'est pas représentatif »

En relatif, il apparaît clairement que les températures mesurées par la fibre dans les zones de mélange (FO TC 8, 10,9 et 5) sont nettement supérieures à celles mesurées par les thermocouples. Ceci est probablement dû à l'effet intégrateur de la fibre : la mesure est une moyenne sur $0.29 \mathrm{~m}$, sur une zone de mélange dont la température varie de manière importante. Par conséquent, la température vue par la fibre est une moyenne de cette zone de mélange et est plus élevée que celle vue par le thermocouple, qui est situé au point le plus froid.

Afin de comparer les deux types de mesures, nous avons choisi de nous concentrer sur la mesure de la température de peau de la canalisation de refroidissement (TC1).

Les figures suivantes (Figure 10 et Figure 11) présentent des zooms sur différentes périodes relativement stables en températures. Sur toutes ces périodes, la mesure par la fibre et celle par thermocouple sont très cohérentes. L'écart entre les deux mesures est de l'ordre de $0.3^{\circ} \mathrm{C}$, ce qui est meilleur que les précisions attendues des deux systèmes. 


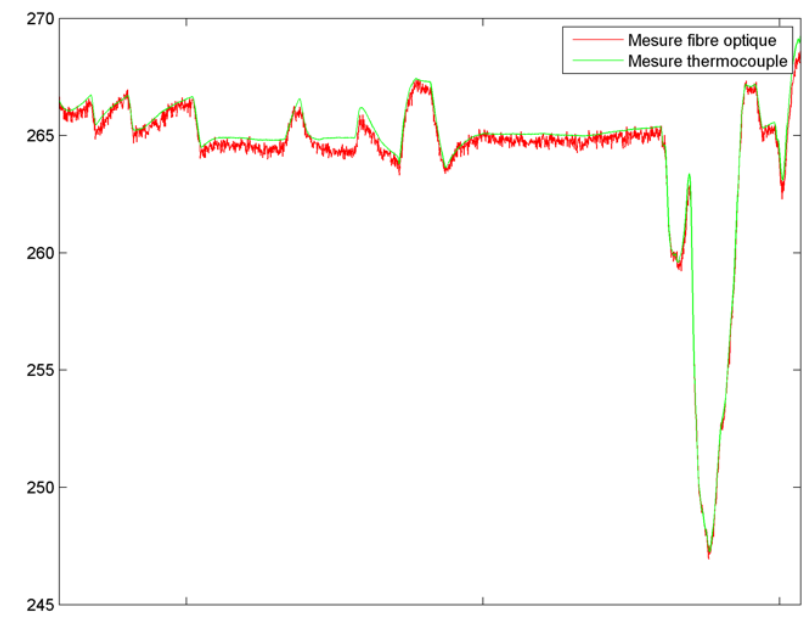

Figure 10. Température par FO et TC : zoom sur une période chaude

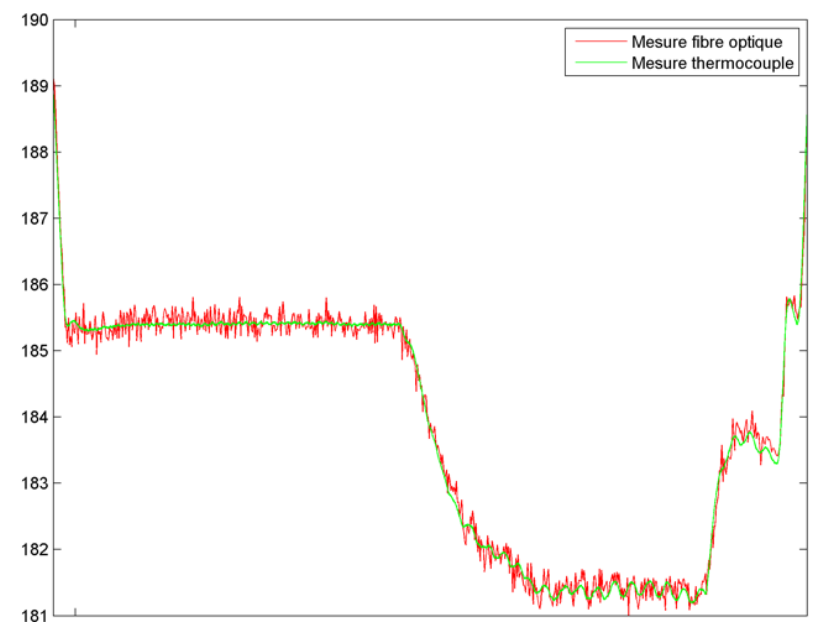

Figure 11. Température par FO et TC : zoom sur une période chaude

\subsubsection{Exploitation 3D}

Nous avons reconstitué la géométrie de pose des câbles fibre optique sous Matlab $®$ afin de pouvoir reconstruire la température de toute la surface de canalisation. La Figure 12 illustre cette géométrie reconstruite.

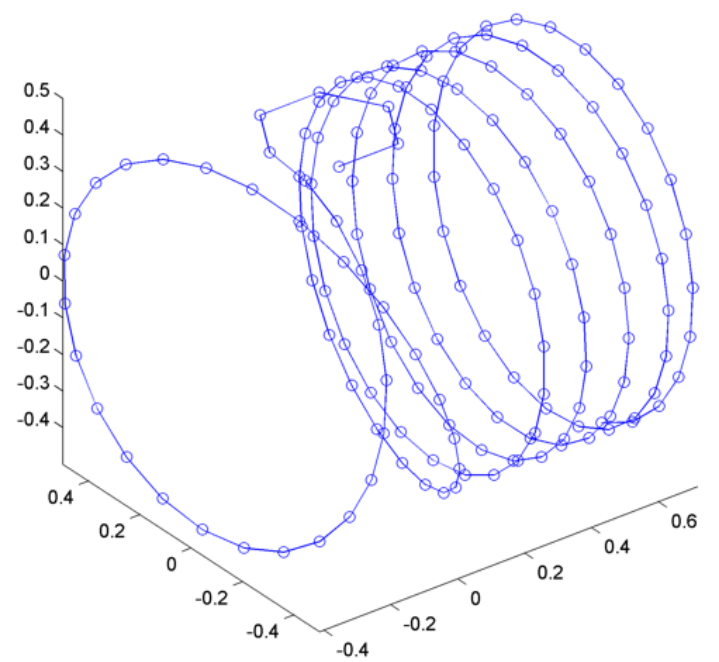

Figure 12. Géométrie reconstruite sous Matlab ${ }^{\circledR}$
Il est alors possible de visualiser les températures mesurées en 3D. Ceci est illustré Figure 13.

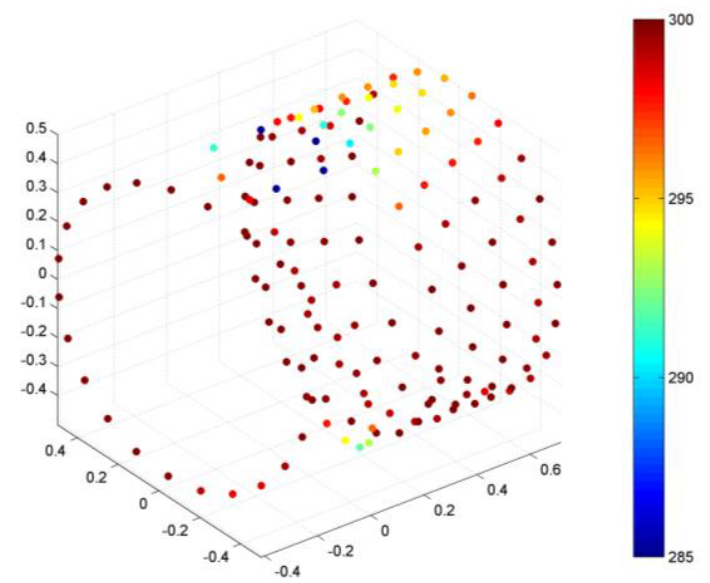

Figure 13. Visualisation 3D des températures mesurées.

A partir de ces données, il est aisé de réaliser une interpolation du champ de température à la surface de la canalisation. Le résultat est présenté en visualisation 3D sur la Figure 14.

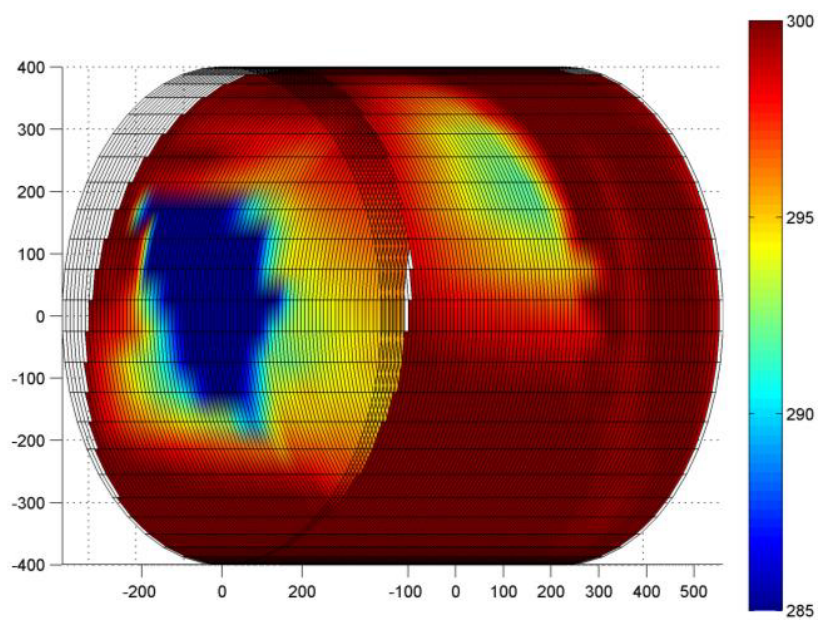

Figure 14. Température de surface, visualisation 3D (dessous)

La zone d'entrée de l'eau froide et la zone de mélange sont nettement visibles sur la gauche de la figure. La deuxième tâche, à droite sur la figure, est due au contact de la fibre avec un support de la canalisation. Ce support est nettement plus froid que la canalisation et crée donc une zone froide par effet de drain thermique.

Bien évidemment cette interpolation n'a de sens que dans les zones où la fibre est suffisamment dense.

\subsection{Ultrasons}

Le calcul de la température à partir des signaux ultrasonores nécessite des traitements permettant d'isoler le signal d'intérêt et de calculer le temps de vol de l'onde ultrasonore au sein du fluide. Un calibrage à froid permet de déterminer le diamètre interne exact de la canalisation, et donc de calculer la célérité de l'onde à partir de ce temps de vol. Enfin, les tables de l'eau permettent de déduire la 
température de la célérité, la pression étant connue (et constante).

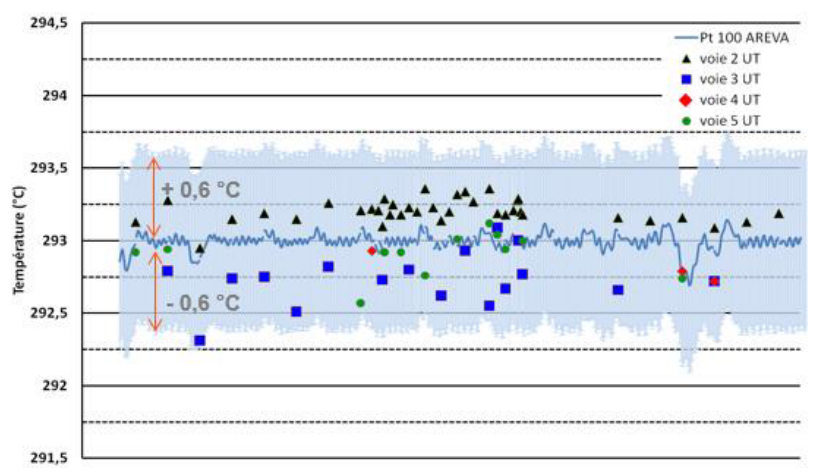

Figure 15. Comparaison ultrasons / sonde interne

La Figure 15 présente la comparaison entre des mesures réalisées à l'aide d'un doigt de gant classique (sonde Pt100) et plusieurs mesures par ultrasons, à diverses positions de la zone de mélange.

Ces premiers résultats permettent de montrer la faisabilité d'une mesure non intrusive de température interne, dans des conditions quasi-industrielles. Pour ce premier essai, l'écart-type expérimental entre la sonde et les différentes voies de mesures des ultrasons est de :

- $\quad \pm 0,2^{\circ} \mathrm{C}$ pour les voies 2 et 3

- $\quad \pm 0,3{ }^{\circ} \mathrm{C}$ pour la voie 4

- $\quad \pm 0,4^{\circ} \mathrm{C}$ pour la voie 5

Les voies 4 et 5 sont situées dans la zone de mélange. La température mesurée est donc une moyenne des températures rencontrées par l'onde ultrasonore au cours de son trajet. Il est donc normal qu'elles soient différentes des températures du doigt de gant qui est situé dans une zone homogène.

\section{Conclusions}

La mesure de température sur la boucle JSPM a permis de valider en environnement industriel équivalent à un CNPE (hors radiation), la faisabilité de la mesure de température par fibre optique et par ultrasons. Les résultats donnés par les instrumentations testées sont de très bonne qualité. Les TC scotchés ont permis de détecter des variations de température de quelques centièmes de degrés en peau externe, équivalentes à des variations de température de quelques dixièmes de degrés à l'intérieur de la canalisation. La fibre optique a permis la reconstruction de la température de la surface externe de la canalisation, avec correction des effets d'atténuation différentielle. Les ultrasons ont permis de mesurer de manière non intrusive des températures moyennes au sein d'une canalisation, même lorsque le fluide est hétérogène en témpérature. Ces résultats permettent d'envisager une utilisation sur CNPE.

\section{Références}

1. J-M Henault, G. Moreau, S. Blairon, J Salin, J-R Courivaud, F Taillade, E Merliot, J-P Dubois, J Bertrand, S Buschaert, S Mayer, S Delepine-Lesoille,
Truly distributed optical fiber sensors for structural health monitoring: From the telecommunication optical fiber drawling tower to water leakage detection in dikes and concrete structure strain monitoring.

2. A Ukil, H Braendle, P Krippner. Distributed Temperature Sensing: Review of Technology and Applications. IEEE SENSORS JOURNAL, VOL. 12, NO. 5, MAY 2012 